\title{
Commentary
}

\section{CMV prophylaxis: a useful step towards prevention of post-transplant diabetes?}

Diabetes is a common complication of organ transplantation, with reported frequencies ranging from 3 to $45 \%$ of renal transplant recipients. The wide range around this estimate reflects differences between immunosuppressive regimens, differing definitions of post-transplant diabetes, and a failure to correct for the background incidence of diabetes mellitus in the transplant population. We analysed 352 renal transplant recipients who received calcineurin inhibitor (ciclosporin A or tacrolimus) and corticosteroid "double therapy" in our unit. We found that $9 \%$ of patients receiving ciclosporin $\mathrm{A}\left(12 \mathrm{mg} \cdot \mathrm{kg}^{-1} \cdot \mathrm{day}^{-1}\right)$ and $11 \%$ of patients receiving tacrolimus $\left(0.2 \mathrm{mg} \cdot \mathrm{kg}^{-1} \cdot \mathrm{day}^{-1}\right)$ developed diabetes within 3 months of transplantation, as defined using the ADA criteria for diagnosis of diabetes, and still had diabetes 1 year after transplantation. This rate of approximately $10 \%$ is consistent with the published data, and confirms that posttransplant diabetes is a common problem. Diabetes has an impact on transplant outcome in terms of lifestyle restrictions and-although definitive studies are lacking - there are indications that patient and transplant survival are both adversely affected $[1,2]$. How then can we minimise the impact of this problem?

Post-transplant diabetes has multiple causes, but the most important is the effect of immunosuppressive drugs on glucose control [3]. Corticosteroids induce insulin resistance by a range of mechanisms including decreased activation of muscle glycogen synthase, reduced insulin receptor density and affinity of insulin binding, and direct effects on the beta cell $[4,5,6,7$, 8]. These effects are exacerbated by calcineurin inhibitors. Ciclosporin A and tacrolimus induce insulin

Received: 21 June 2004 / Accepted: 28 July 2004

Published online: 25 August 2004

(C) Springer-Verlag 2004 resistance and reduce beta cell insulin mRNA levels, beta cell insulin content and in vitro and in vivo insulin release $[9,10,11,12,13]$. Long-term exposure to the combination of calcineurin inhibitors and corticosteroids will lead to beta cell death in sensitive recipients. In an ideal world we would avoid these diabetogenic agents, but alternative therapies (see Table 1) are unfortunately still poorly validated for use in the first 3 months after transplantation. This is because the number of patients exposed remains small, because they have typically been used alongside rather than in place of calcineurin inhibitors, and because long-term follow up is lacking. The dilemma is at its most acute with islet transplantation, which has been revolutionised by the success of the Edmonton protocol $[14,15]$. A major factor in its success is preservation of beta cell viability and function, and the use of non-diabetogenic immunosuppressive therapy has played a key role in this. Corticosteroids are avoided but tacrolimus was considered necessary, albeit at a low dose, while corticosteroids and calcineurin inhibitors continue to be used in whole pancreas transplantation. In other words we still do not have enough confidence in non-diabetogenic immunosuppressive regimens for whole-scale changes in primary immunosuppression.

How then might we reduce the incidence of posttransplant diabetes? Most patients who receive a solid organ transplant are exposed to calcineurin inhibitors and oral corticosteroids, yet only approximately $10 \%$ develop post-transplant diabetes. This suggests that it may be possible to focus the use of novel immunosuppressive strategies on patients in whom the risk of post-transplant diabetes is considered to be high. This approach soon runs into problems, however. To begin with, more than 20 different risk factors are proposed in the 25 studies that have reported risk factors for development of post-transplant diabetes. Of these, only 
Table 1. Immunosuppressive agents and diabetes

\begin{tabular}{lll}
\hline Drug & Diabetogenic & Licensed for primary immunosuppression \\
\hline Ciclosporin A & +++ & Yes \\
Tacrolimus & +++ & Yes \\
Corticosteroids & +++ & Yes \\
Rapamycin & +- & In combination with CnI and corticosteroids \\
Mycophenolate mofetil & - & In combination with CnI and corticosteroids \\
Daclizumab/Basiliximab & - & In combination with CnI and corticosteroids \\
Everolimus & Probably not & Licensed in Europe (but not in UK) for use in combination \\
FTY 720 & Probably not & with CnI and corticosteroids \\
Myfortic & Probably not & In combination with ciclosporin and corticosteroids \\
\hline
\end{tabular}

+++, markedly; +/-, possibly; CnI, calcineurin inhibitor

increasing age and African-American or Hispanic, as opposed to European, extraction are generally agreed to be associated with a higher incidence of diabetes (reviewed in [16]). Familiar risk factors for Type 1 or Type 2 diabetes, such as weight, family history of diabetes, and the HLA susceptibility alleles for Type 1 diabetes (DR3, DR4 and DQ2), do not feature consistently, underlining the separate aetiology of posttransplant diabetes, and demonstrating that reliable identification of at-risk patients prior to transplantation is not possible.

Clinically apparent CMV infection has also been identified as a risk factor for development of posttransplant diabetes. This is of particular interest because CMV infection can be prevented. The report by Hjelmesæth et al. in this edition extends these observations by suggesting that clinically silent CMV infection is also a risk factor [17]. The patients studied formed part of a cohort of 173 consecutive non-diabetic renal transplant recipients. Post-transplant dia-betes mellitus was diagnosed using the OGTT, and only patients receiving ciclosporin-A-based triple immunosuppression who did not develop clinically significant CMV disease were included. Of 124 patients fulfilling these criteria, 63 did not develop CMV infection and 61 developed asymptomatic CMV infection as diagnosed by demonstration of CMV pp65 antigenaemia.

Clinical CMV disease is associated with significant morbidity and mortality. The European Best Practice Guidelines (EBPG) [18] acknowledge evidence supporting the use of a wide range of prophylactic therapies, of varying efficacy, in order to minimise the incidence of this significant complication of immunosuppressive therapy. With low-dose calcineurin-inhibitor-based double or triple therapy, as currently given to most solid organ transplant recipients, the incidence of CMV disease is low, and prophylaxis aimed at preventing clinically significant disease is only recommended in seronegative recipients of transplants from seropositive donors. If additional immunosuppression is used (ATG, ALG or OKT3), prophylaxis is then recommended also for seropositive-seropositive com- binations. Pharmacoeconomic analyses conclude that CMV prophylaxis in donor positive-recipient negative combinations is the current preferred strategy [19], but a recent analysis called for further trials to establish which prophylactic strategy is best [20]. These analyses consider only clinically significant CMV disease. The EBPG suggest that treatment of asymptomatic disease, detected by screening, is advisable to prevent spread of CMV infection and clinically significant disease. The suggestion that asymptomatic CMV infection predisposes to post-transplant diabetes adds an important new facet to this debate. Routine screening for asymptomatic CMV infection is not undertaken in most solid organ transplant recipients and analyses have not considered prevention of asymptomatic disease an important goal.

This relatively small report requires confirmation, but nonetheless raises the possibility that CMV prophylaxis may be a useful and cost-effective strategy for management of post-transplant diabetes. It should therefore prompt more detailed analysis of the role of CMV infection in the development of post-transplant diabetes, should remind us of the importance of nonimmunological factors as determinants of transplant outcome, and should keep alive the debate about CMV prophylaxis in solid organ transplantation.

R. M. Smith

Academic Renal Unit, University of Bristol, UK

R. M. Smith

The Richard Bright Renal Unit, Southmead Hospital, Bristol, UK

\section{References}

1. Jindal RM, Hjelmesæth J (2000) Impact and management of posttransplant diabetes mellitus. Transplantation 70 [Suppl]:SS58-SS63

2. Revanur VK, Jardine AG, Kingsmore DB, Jaques BC, Hamilton DH, Jindal RM (2001) Influence of diabetes mellitus on patient and graft survival in recipients of kidney transplantation. Clin Transplant 15:89-94 
3. Weir MR, Fink JC (1999) Risk for posttransplant diabetes mellitus with current immunosuppressive medications. Am J Kidney Dis 34:1-13

4. Ekstrand AV (1991) Effect of steroid-therapy on insulin sensitivity in insulin-dependent diabetic patients after kidney transplantation. J Diabetic Complications 5:244-248

5. Ekstrand AV, Eriksson JG, Gronhagen-Riska C, Ahonen PJ, Groop LC (1992) Insulin resistance and insulin deficiency in the pathogenesis of posttransplantation diabetes in man. Transplantation 53:563-569

6. Ekstrand A, Schalin-Jantti C, Lofman M et al. (1996) The effect of (steroid) immunosuppression on skeletal muscle glycogen metabolism in patients after kidney transplantation. Transplantation 61:889-893

7. Kahn CR, Goldfine ID, Neville DM et al. (1978) Alteration in insulin binding induced by changes in vivo in the levels of glucocorticoids and growth hormone. Endocrinology 103:1054-1066

8. Rizza MA, Mandarino LJ, Gerich JE (1982) Cortisolinduced insulin resistance in man: impaired suppression of glucose production and stimulation of glucose utilization due to a post-receptor defect of insulin action. J Clin Endocrinol Metab 54:131-138

9. Ishizuka J, Gugliuzza KK, Wassmuth Z et al. (1993) Effects of FK506 and cyclosporine on dynamic insulin secretion from isolated dog pancreatic islets. Transplantation 56:1486-1490

10. Polastri L, Galbiati F, Bertuzzi F et al. (2002) Secretory defects induced by immunosuppressive agents on human pancreatic beta-cells. Acta Diabetol 39:229-233

11. Redmon JB, Olson LK, Armstrong MB, Greene MJ, Robertson RP (1996) Effects of tacrolimus (FK506) on human insulin gene expression, insulin mRNA levels, and insulin secretion in HIT-T15 cells. J Clin Invest 98:2786-2793

12. Robertson RP (1986) Cyclosporin-induced inhibition of insulin secretion in isolated rat islets and HIT cells. Diabetes 35:1016-1019
13. Strasser S, Alejandro R, Shapiro ET, Ricordi C, Todo S, Mintz DH (1992) Effect of FK506 on insulin secretion in normal dogs. Metabolism 41:64-67

14. Ryan EA, Lakey JR, Paty BW et al. (2002) Successful islet transplantation: continued insulin reserve provides longterm glycemic control. Diabetes 51:2148-2157

15. Shapiro AM, Lakey JR, Ryan EA et al. (2000) Islet transplantation in seven patients with type 1 diabetes mellitus using a glucocorticoid-free immunosuppressive regimen. N Engl J Med 343:230-238

16. Marchetti P (2001) Strategies for risk reduction and management of posttransplant diabetes mellitus. Transplant Proc 33 [Suppl 5A]:27S-31S

17. Hjelmesæth J, Sagedal S, Hartmann A et al. (2004) Asymptomatic cytomegalovirus infection is associated with increased risk of new-onset diabetes mellitus and impaired insulin release after renal transplantation. Diabetologia 47 DOI 10.1007/s00125-004-1499-z

18. EBPG Expert Group on Renal Transplantation (2000) European Best Practice Guidelines for Renal Transplantation (Part 1). Nephrol Dial Transplant 15 [Suppl 7]:7174

19. Mauskopf JA, Richter A, Annemans L, Maclaine G (2000) Cost-effectiveness model of cytomegalovirus management strategies in renal transplantation. Comparing valacyclovir prophylaxis with current practice. Pharmacoeconomics 18:239-251

20. Das A (2003) Cytomegalovirus infection in solid organ transplantation: economic implications. Pharmacoeconomics 21:467-475

R. M. Smith (『)

The Richard Bright Renal Unit, Southmead Hospital, Bristol, BS10 5NB, UK

E-mail: Richard.Smith@bristol.ac.uk

Tel.: +44-117-9595438, Fax: +44-117-9595438 